\title{
DIRICHLET POLYHEDRA FOR SIMPLEX GROUPS OF SPHERICAL, EUCLIDEAN OR HYPERBOLIC SPACES
}

\author{
Akira Ushijima \\ Kanazawa University, Faculty of Mathematics and Physics, Institute of Science and Engineering \\ Ishikawa 920-1192, Japan; ushijima@se.kanazawa-u.ac.jp
}

\begin{abstract}
Komori and Umemoto detected combinatorial shapes of Dirichlet polyhedra for simplex groups acting on hyperbolic space. We generalize their result to simplex groups acting on Euclidean or spherical spaces.
\end{abstract}

\section{Introduction}

Several methods are known to decompose manifolds into simpler pieces. For manifolds obtained as the quotient space of a finitely compact metric space by the action of a discrete group of isometries, Dirichlet polyhedra are one such decomposition. One of the reasons why Dirichlet polyhedra are so important for studying such manifolds is that they provide combinatorial representations of discrete groups.

In general, it is not obvious to determine Dirichlet polyhedra explicitly. For constructing a Dirichlet polyhedron, we need to choose not only a discrete group but also a point in the metric space, which will be called the center of the Dirichlet polyhedron. Take the set of points which are the images of the center under the action of the group. The Dirichlet polyhedron is then defined by the Voronoi tessellation with respect to this set: it is the Voronoi cell containing the center. Since the Voronoi tessellation is determined by infinitely many points, it is not obvious in general to determine the explicit geometric shape of Dirichlet polyhedron, even in the case of finitely sided polyhedron.

For a given discrete group, we focus on possible combinatorial shapes of Dirichlet polyhedra. Though each Dirichlet polyhedron is determined by the group together with the center, possible combinatorial shapes depend only on the group. As we have mentioned before, it is difficult to determine Dirichlet polyhedra for a general discrete group. There are, however, specialized cases where the combinatorial shapes of Dirichlet polyhedra are known. A discrete group of orientation-preserving isometries acting on a two-dimensional hyperbolic space is called a Fuchsian group. Näätänen determined in [Nää85] combinatorial shapes of Dirichlet polyhedra (or more precisely Dirichlet polygon in this case) for a Fuchsian group which yields a particularly symmetric genus two closed orientable surface as its quotient space. Other examples were provided by Umemoto in [Ume11] for Fuchsian groups that are index two subgroups of triangle groups. Komori and Umemoto announced in [KU11] that Umemoto's results are able to generalize to higher-dimensional hyperbolic space.

Based on Komori and Umemoto's previous studies, what we will prove here is that their results also hold for Euclidean or spherical reflection groups.

Acknowledgements. Umemoto's result [Ume11] was suggested to the author by Professor Kazuhiro Ichihara, to whom the author wants to express his gratitude first

https://doi.org/10.5186/aasfm.2021.4607

2020 Mathematics Subject Classification: Primary 57S30, 51F15.

Key words: Simplex group, Dirichlet polyhedron. 
of all. The author also wants to express his gratitude to Professors Yohei Komori and Yuriko Umemoto for sharing their result and related topics, in particular about [KU11]. I finally would like to thank the referees for their valuable suggestions for improving the original manuscript.

\section{Preliminaries}

For a positive integer $n$, we use the symbol $\mathbf{X}^{n}$ to denote either the $n$-dimensional spherical, Euclidean or hyperbolic spaces. Let $\rho$ and $\mu$ be the distance function and the volume function on $\mathbf{X}^{n}$ respectively. Let $I_{n}$ be the set $\{1,2, \ldots, n\}$ consisting of $n$ integers. For $k \in I_{n}$, a $k$-dimensional plane in $\mathbf{X}^{n}$ is a subset of $\mathbf{X}^{n}$ that is isometric to $\mathbf{X}^{k}$. In particular, $(n-1)$-dimensional planes are called hyperplanes. For a non-empty subset $S \subset \mathbf{X}^{n}$, we denote by $\langle S\rangle$ the plane in $\mathbf{X}^{n}$ which contains $S$ and which is minimal with respect to dimension. We also denote by $\partial S$ and $\operatorname{Int}(S)$ the boundary and the interior of $S$ in $\langle S\rangle$ with respect to its subspace topology, respectively.

For $k \in I_{n}$, points $v_{1}, v_{2}, \ldots, v_{k+1} \in \mathbf{X}^{n}$ are said to be in a general position if they are affinely independent, that is, no $(k-1)$-dimensional plane in $\mathbf{X}^{n}$ contains them. A single point is always regarded in a general position. For points $v_{1}, v_{2}$, $\ldots, v_{k+1} \in \mathbf{X}^{n}$ in a general position, we denote by $v_{1} v_{2} \cdots v_{k+1}$ the $k$-dimensional simplex, or simply $k$-simplex, in $\mathbf{X}^{n}$. In other words, $v_{1} v_{2} \cdots v_{k+1}$ is the convex hull for $v_{1}, v_{2}, \ldots, v_{k+1}$ in $\mathbf{X}^{n}$. The points $v_{1}, v_{2}, \ldots, v_{k+1}$, which are also regarded as 0 -dimensional simplexes, are called the vertices of $v_{1} v_{2} \cdots v_{k+1}$. The join of two simplexes is denoted by the binary operator $*$.

Let $\operatorname{Isom}^{+}\left(\mathbf{X}^{n}\right)$ be the group of all orientation-preserving isometries on $\mathbf{X}^{n}$. We denote by $\iota \in \operatorname{Isom}^{+}\left(\mathbf{X}^{n}\right)$ the identity mapping. For any $f \in \operatorname{Isom}^{+}\left(\mathbf{X}^{n}\right)$, let $\operatorname{Fix}(f)$ be the set of all fixed points by the action of $f$ on $\mathbf{X}^{n}$ :

$$
\operatorname{Fix}(f):=\left\{p \in \mathbf{X}^{n} \mid f(p)=p\right\} .
$$

For $f \in \operatorname{Isom}^{+}\left(\mathbf{X}^{n}\right)$ and for $p \in \mathbf{X}^{n}$, let $\mathrm{H}_{p}(f)$ be a closed half-space defined as follows:

$$
\mathrm{H}_{p}(f):=\left\{q \in \mathbf{X}^{n} \mid \rho(q, p) \leq \rho(q, f(p))\right\} .
$$

Let $G$ be a discrete subgroup of $\operatorname{Isom}^{+}\left(\mathbf{X}^{n}\right)$. As in [Rat06, §6.7], we define the Dirichlet polyhedron $\mathrm{D}_{p}(G)$ of $G$ with center $p \in \mathbf{X}^{n}-\bigcup_{f \in G-\{\iota\}} \operatorname{Fix}(f)$ as

$$
\mathrm{D}_{p}(G):=\bigcap_{f \in G-\{\iota\}} \mathrm{H}_{p}(f) .
$$

It is a locally finite convex fundamental polyhedron for $G$.

For an $n$-simplex $\Delta:=v_{1} v_{2} \ldots v_{n+1}$ in $\mathbf{X}^{n}$, let

$$
\Delta_{i}:=\Delta \cap\left\langle\left\{v_{1}, v_{2}, \ldots, v_{n+1}\right\}-\left\{v_{i}\right\}\right\rangle
$$

be the $(n-1)$-dimensional simplex which is opposite in $\Delta$ to the vertex $v_{i}$, and let $R_{i}$ be the reflection of $\mathrm{X}^{n}$ along the hyperplane $\left\langle\Delta_{i}\right\rangle$. From now on, $\Delta$ is assumed to yield a simplex group $\Gamma_{\Delta}$ defined in [KU11] and in [Rat06, §7.2]. That is, $\Gamma_{\Delta}$ is a discrete subgroup of $\operatorname{Isom}^{+}\left(\mathbf{X}^{n}\right)$ generated by all transformations of the form $R_{i} \circ R_{j}$ for any $i, j \in I_{n+1}$.

We study combinatorial shapes of Dirichlet polyhedra for $\Gamma_{\Delta}$ with respect to centers. For our purpose, points in $\operatorname{Int}(\Delta) \sqcup \bigsqcup_{i \in I_{n+1}} \operatorname{Int}\left(\Delta_{i}\right)$ are enough to be taken as centers, since the images of the closure of $\operatorname{Int}(\Delta) \sqcup \bigsqcup_{i \in I_{n+1}} \operatorname{Int}\left(\Delta_{i}\right)$ by the action of 
$\Gamma_{\Delta}$ gives a tessellation of $\mathbf{X}^{n}$. Furthermore, the set of points in $\Delta$ that are not fixed by any non-trivial elements of $\Gamma_{\Delta}$ is disjointly decomposed as follows:

$$
\Delta-\bigcup_{f \in \Gamma_{\Delta}-\{\iota\}} \operatorname{Fix}(f)=\operatorname{Int}(\Delta) \sqcup \bigsqcup_{i \in I_{n+1}} \operatorname{Int}\left(\Delta_{i}\right),
$$

since each generator $R_{i} \circ R_{j} \in \Gamma_{\Delta}$ is a rotation along the plane $\left\langle\Delta_{i} \cap \Delta_{j}\right\rangle$ of codimension two, and since $\partial \Delta_{i} \subset \bigcup_{j \in I_{n+1}-\{i\}}\left\langle\Delta_{i} \cap \Delta_{j}\right\rangle$. Based on these observations, the following theorem is enough to detect combinatorial shapes of Dirichlet polyhedra for $\Gamma_{\Delta}$ :

Theorem. Let $\Gamma_{\Delta}$ be a discrete simplex group defined by an $n$-simplex $\Delta$. The Dirichlet polyhedron $\mathrm{D}_{p}\left(\Gamma_{\Delta}\right)$ of $\Gamma_{\Delta}$ centered at a point $p$ is given as follows:

$$
\mathrm{D}_{p}\left(\Gamma_{\Delta}\right)= \begin{cases}\bigcap_{i, j \in I_{n+1}, i \neq j} \mathrm{H}_{p}\left(R_{i} \circ R_{j}\right), & \text { for } p \in \operatorname{Int}(\Delta), \\ \bigcap_{j \in I_{n+1}-\{i\}}\left(\mathrm{H}_{p}\left(R_{i} \circ R_{j}\right) \cap \mathrm{H}_{p}\left(R_{j} \circ R_{i}\right)\right), & \text { for } p \in \operatorname{Int}\left(\Delta_{i}\right),\end{cases}
$$

where $\Delta_{i}$ is the $(n-1)$-dimensional simplex which is opposite in $\Delta$ to the vertex $v_{i}$, $R_{i}$ is the reflection of $\mathbf{X}^{n}$ along the hyperplane $\left\langle\Delta_{i}\right\rangle$ containing $\Delta_{i}$, and $\mathrm{H}_{p}\left(R_{i} \circ R_{j}\right)$ is the closed half-space defined as $\mathrm{H}_{p}\left(R_{i} \circ R_{j}\right):=\left\{q \in \mathbf{X}^{n} \mid \rho(q, p) \leq \rho\left(q, R_{i} \circ R_{j}(p)\right)\right\}$.

Remark. When $\mathbf{X}^{2}$ is the hyperbolic plane, the same result has already been obtained by [Ume11]. In [KU11], Komori and Umemoto announced that the same result holds when $\mathbf{X}^{n}$ is the $n$-dimensional hyperbolic space. Furthermore, they also mentioned that the same result holds when vertices of $\Delta$ are ideal.

\section{Proof of Theorem}

We start by introducing the notion for dihedral angles of $\Delta$, which will be used to state the following lemma of a higher-dimensional generalization of Ceva's theorem. For a subset $S$ in $\mathbf{X}^{n}$ bounded by two transversely intersecting hyperplanes, we denote by $\theta(S)$ its dihedral angle. In particular, for $e \in v_{i} v_{j}$, we also denote by $\theta\left(v_{j} e\right)$ the dihedral angle in $\Delta$ which is opposite to the 1 -simplex $e v_{j}$. That is, the dihedral angle between the hyperplanes $\left\langle\left\{v_{1}, v_{2}, \ldots, v_{n+1}, e\right\}-\left\{v_{i}, v_{j}\right\}\right\rangle$ and $\left\langle\left\{v_{1}, v_{2}, \ldots, v_{n+1}, e\right\}-\left\{v_{i}, e\right\}\right\rangle$. The composition $R_{i} \circ R_{j}$ for any mutually distinct $i$, $j \in I_{n+1}$ is a rotation from $\left\langle\Delta_{j}\right\rangle$ toward $\left\langle\Delta_{i}\right\rangle$ along $\left\langle\Delta_{i} \cap \Delta_{j}\right\rangle$, which is opposite to $v_{i} v_{j}$ in $\Delta$. So we have

$$
\left.\theta\left(\mathrm{H}_{j} \cap\left(R_{i} \circ R_{j}\right)\left(R_{j}\left(\mathrm{H}_{j}\right)\right)\right)=\theta\left(\mathrm{H}_{j} \cap R_{i}\left(\mathrm{H}_{j}\right)\right)\right)=2 \theta\left(v_{i} v_{j}\right),
$$

where $\mathrm{H}_{j}:=\mathrm{H}_{v_{j}}\left(R_{j}\right)$, meaning that $\mathrm{H}_{j}$ is the closed halfspace supported by $\left\langle\Delta_{j}\right\rangle$ and containing $\Delta$.

Lemma. [Ush19, Theorem 3] For an $n$-simplex $\Delta$ and points $e_{i, j}=e_{j, i} \in v_{i} v_{j}$ for any $i, j \in I_{n+1}$, the intersection of $(n-1)$-simplexes $v_{i_{1}} \ldots v_{i_{n-1}} e_{k, m}$, where indexes are taken all possible choices satisfying $\left\{i_{1}, \ldots, i_{n-1}, k, m\right\}=I_{n+1}$, consists of a point in $\Delta$ if and only if the equation

$$
\frac{\sin \theta\left(v_{i} e_{i, j}\right) \sin \theta\left(v_{j} e_{j, k}\right) \sin \theta\left(v_{k} e_{k, i}\right)}{\sin \theta\left(v_{i} e_{i, k}\right) \sin \theta\left(v_{k} e_{k, j}\right) \sin \theta\left(v_{j} e_{j, i}\right)}=1
$$

holds for any mutually distinct $i, j, k \in I_{n+1}$. 
The key fact used in the proof of [Ume11, Theorem 24] is this lemma in the case that $\mathbf{X}^{n}$ is the hyperbolic plane, which is also known as Ceva's theorem for the hyperbolic plane. If we want to prove a generalization of Umemoto's result for discrete simplex groups in $\operatorname{Isom}^{+}\left(\mathbf{X}^{n}\right)$ following the strategy of her proof, we need to prepare a corresponding generalization of Ceva's theorem, that is this lemma.

Proof of Theorem. To obtain (1), it is enough to show that the sets on each side of the equation have equal volumes. Note that by definition, the Dirichlet polyhedron $\mathrm{D}_{p}\left(\Gamma_{\Delta}\right)$ is a subset of each set appearing on the right-hand side of (1). Since all sets in question are constructed as the intersection of closed halfspaces, if $\mathrm{D}_{p}\left(\Gamma_{\Delta}\right)$ is a proper subset, then its volume must be strictly less than the volume of the sets appearing on the right-hand side of (1). If the volumes are equal, however, then the sets on each side of (1) must coincide.

The volume $\mu\left(\mathrm{D}_{p}\left(\Gamma_{\Delta}\right)\right)$ is computed as follows. The fundamental regions $\mathrm{D}_{p}\left(\Gamma_{\Delta}\right)$ and $\Delta \cup R_{i}(\Delta)$ are proper in the sense that the volumes of their boundary are zero. Thus they have the same volume by [Rat06, Theorem 6.7.2], which implies

$$
\mu\left(\mathrm{D}_{p}\left(\Gamma_{\Delta}\right)\right)=\mu\left(\Delta \cup R_{i}(\Delta)\right)=\mu(\Delta)+\mu\left(R_{i}(\Delta)\right)=2 \mu(\Delta) .
$$

The aim of our proof thus comes to show that the volumes of the sets on the righthand side of $(1)$ are $2 \mu(\Delta)$.

For $p \in \operatorname{Int}\left(\Delta_{i}\right)$ for any $i \in I_{n+1}$, we actually have

$$
\bigcap_{j \in I_{n+1}-\{i\}}\left(\mathrm{H}_{p}\left(R_{i} \circ R_{j}\right) \cap \mathrm{H}_{p}\left(R_{j} \circ R_{i}\right)\right)=\Delta \cup R_{i}(\Delta),
$$

since $\mathrm{H}_{p}\left(R_{j} \circ R_{i}\right)=\mathrm{H}_{j}, \mathrm{H}_{p}\left(R_{i} \circ R_{j}\right)=R_{i}\left(\mathrm{H}_{j}\right)$, and since $R_{i}\left(\mathrm{H}_{j}\right)$ is a halfspace supported by $\left\langle R_{i}\left(\Delta_{j}\right)\right\rangle$ and containing $R_{i}(\Delta)$.

We will show that $\mu\left(\bigcap_{i, j \in I_{n+1}, i \neq j} \mathrm{H}_{p}\left(R_{i} \circ R_{j}\right)\right)=2 \mu(\Delta)$ for any $p \in \operatorname{Int}(\Delta)$ from now on. We first study the shape of $R_{i}\left(\mathrm{H}_{i}\right) \cap \bigcap_{j \in I_{n+1}-\{i\}} \mathrm{H}_{p}\left(R_{i} \circ R_{j}\right)$. As we have seen at the beginning of this section, $R_{i} \circ R_{j}$ is the rotation along $\left\langle\Delta_{i} \cap \Delta_{j}\right\rangle$ with rotation angle $2 \theta\left(v_{i} v_{j}\right)$ from $\left\langle\Delta_{j}\right\rangle$ toward $\left\langle\Delta_{i}\right\rangle$. So we have

$$
R_{i}\left(\mathrm{H}_{i}\right) \cap \mathrm{H}_{p}\left(R_{i} \circ R_{j}\right) \subset R_{i}\left(\mathrm{H}_{i}\right) \cap R_{i}\left(\mathrm{H}_{j}\right)=R_{i}\left(\mathrm{H}_{i} \cap \mathrm{H}_{j}\right)
$$

for any $j \in I_{n+1}-\{i\}$. This implies that the set in question is an $n$-dimensional pyramid with base $R_{i}\left(\Delta_{i}\right)=\Delta_{i}$ and apex, say, $u_{i}$ in $R_{i}(\Delta)$.

Showing the coincidence of the points $R_{1}\left(u_{1}\right), R_{2}\left(u_{2}\right), \ldots, R_{n+1}\left(u_{n+1}\right)$ is enough to complete the proof by the following reason. We have already shown that

$$
R_{i}\left(\mathrm{H}_{i}\right) \cap \bigcap_{j \in I_{n+1}-\{i\}} \mathrm{H}_{p}\left(R_{i} \circ R_{j}\right)=u_{i} * \Delta_{i} .
$$

This means

$$
\bigcap_{i, j \in I_{n+1}, i \neq j} \mathrm{H}_{p}\left(R_{i} \circ R_{j}\right)=\Delta \cup \bigcup_{i \in I_{n+1}}\left(u_{i} * \Delta_{i}\right)
$$

So we have

$$
\begin{aligned}
\mu\left(\bigcap_{i, j \in I_{n+1}, i \neq j} \mathrm{H}_{p}\left(R_{i} \circ R_{j}\right)\right) & =\mu\left(\Delta \cup \bigcup_{i \in I_{n+1}}\left(u_{i} * \Delta_{i}\right)\right)=\mu(\Delta)+\sum_{i=1}^{n+1} \mu\left(u_{i} * \Delta_{i}\right) \\
& =\mu(\Delta)+\sum_{i=1}^{n+1} \mu\left(R_{i}\left(u_{i}\right) * \Delta_{i}\right) .
\end{aligned}
$$


Since each $R_{i}\left(u_{i}\right) * \Delta_{i}$ is an $n$-dimensional pyramid contained in $\Delta$ with base $\Delta_{i}$ for any $i \in I_{n+1}$, if the points in question coincide with each other, then $\sum_{i=1}^{n+1} \mu\left(R_{i}\left(u_{i}\right) * \Delta_{i}\right)$ must be $\mu(\Delta)$ and the proof is complete.

In order to show the coincidence of $R_{1}\left(u_{1}\right), R_{2}\left(u_{2}\right), \ldots, R_{n+1}\left(u_{n+1}\right)$, we first see that $\left\langle R_{i}\left(u_{i}\right) *\left(\Delta_{i} \cap \Delta_{j}\right)\right\rangle$ coincides with $\left\langle R_{j}\left(u_{j}\right) *\left(\Delta_{i} \cap \Delta_{j}\right)\right\rangle$ for any mutually distinct $i, j \in I_{n+1}$. Since $u_{i} \in \partial \mathrm{H}_{p}\left(R_{i} \circ R_{j}\right)$, we have $R_{i}\left(u_{i}\right) \in \partial R_{i}\left(\mathrm{H}_{p}\left(R_{i} \circ R_{j}\right)\right)$. We also have $\Delta_{i} \cap \Delta_{j} \subset \partial R_{i}\left(\mathrm{H}_{p}\left(R_{i} \circ R_{j}\right)\right)$. So we have $\left\langle R_{i}\left(u_{i}\right) *\left(\Delta_{i} \cap \Delta_{j}\right)\right\rangle=\partial R_{i}\left(\mathrm{H}_{p}\left(R_{i} \circ R_{j}\right)\right)$. Let $e_{i, j}=e_{j, i}$ be the point defined as the intersection between $v_{i} v_{j}$ and the hyperplane $\left\langle\left\{v_{1}, v_{2}, \ldots, v_{n+1}, p\right\}-\left\{v_{i}, v_{j}\right\}\right\rangle$. Then we have

$$
\theta\left(\mathrm{H}_{i} \cap R_{i}\left(\mathrm{H}_{p}\left(R_{i} \circ R_{j}\right)\right)\right)=\theta\left(R_{i}\left(\mathrm{H}_{i}\right) \cap \mathrm{H}_{p}\left(R_{i} \circ R_{j}\right)\right)=\theta\left(e_{i, j} v_{i}\right),
$$

and similarly

$$
\begin{aligned}
\left\langle R_{j}\left(u_{j}\right) *\left(\Delta_{i} \cap \Delta_{j}\right)\right\rangle & =\partial R_{j}\left(\mathrm{H}_{p}\left(R_{i} \circ R_{j}\right)\right), \\
\theta\left(\mathrm{H}_{j} \cap R_{j}\left(\mathrm{H}_{p}\left(R_{j} \circ R_{i}\right)\right)\right) & =\theta\left(e_{j, i} v_{j}\right)
\end{aligned}
$$

hold. So we have

$$
\begin{aligned}
\theta\left(\mathrm{H}_{i} \cap R_{i}\left(\mathrm{H}_{p}\left(R_{i} \circ R_{j}\right)\right)\right)+\theta\left(\mathrm{H}_{j} \cap R_{j}\left(\mathrm{H}_{p}\left(R_{j} \circ R_{i}\right)\right)\right) & =\theta\left(e_{i, j} v_{i}\right)+\theta\left(e_{j, i} v_{j}\right) \\
& =\theta\left(\mathrm{H}_{i} \cap \mathrm{H}_{j}\right),
\end{aligned}
$$

which implies the coincidence of $\partial R_{i}\left(\mathrm{H}_{p}\left(R_{i} \circ R_{j}\right)\right)$ and $\partial R_{j}\left(\mathrm{H}_{p}\left(R_{j} \circ R_{i}\right)\right)$.

Since the vertices in question lie on hyperplanes $\left\langle R_{i}\left(u_{i}\right) *\left(\Delta_{i} \cap \Delta_{j}\right)\right\rangle$, for showing the coincidence of the vertices, it is enough to show that these hyperplanes intersect at one point. In order to do it, let $d_{i, j}=d_{j, i}$ be the point of the intersection of the 1-simplex $v_{i} v_{j}$ and the hyperplane $\left\langle R_{i}\left(u_{i}\right) *\left(\Delta_{i} \cap \Delta_{j}\right)\right\rangle=\left\langle R_{j}\left(u_{j}\right) *\left(\Delta_{i} \cap \Delta_{j}\right)\right\rangle$. Then, by the Lemma, it is enough to show the following equation:

$$
\frac{\sin \theta\left(v_{i} d_{i, j}\right) \sin \theta\left(v_{j} d_{j, k}\right) \sin \theta\left(v_{k} d_{k, i}\right)}{\sin \theta\left(v_{i} d_{i, k}\right) \sin \theta\left(v_{k} d_{k, j}\right) \sin \theta\left(v_{j} d_{j, i}\right)}=1 .
$$

Since $\theta\left(v_{i} d_{i, j}\right)=\theta\left(v_{j} e_{i, j}\right)$, the desired equation holds, again, by the Lemma for the center $p \in \Delta$ of $\mathrm{D}_{p}\left(\Gamma_{\Delta}\right)$.

\section{References}

[Bea83] Beardon, A. F.: The geometry of discrete groups. - Grad. Texts in Math. 91, SpringerVerlag, New York, 1983.

[KU11] Komori, Y., and Y. Umeмото: On Dirichlet polyhedra for generalized simplex groups. - In: Proceedings of MSJ Autumn Meeting, Complex Analysis Section, 2011, 20.

[Nää85] NÄÄtÄNEN, M.: On the stability of identification patterns for Dirichlet regions. - Ann. Acad. Sci. Fenn. Ser. A I Math. 10, 1985, 411-417.

[Rat06] Ratcliffe, J. G.: Foundations of hyperbolic manifolds. - Grad. Texts in Math. 149, Springer, New York, second edition, 2006.

[Ume11] Uмемото, Y.: On Dirichlet fundamental domains for Fuchsian groups. - Master's thesis, Osaka City University, Osaka, 2011.

[Ush19] Ushijima, A.: Ceva's and Menelaus' theorems for higher-dimensional simplexes. - J. Geom. 110, 2019, 13. 